\title{
Brain MR Imaging of Patients with Perinatal Chikungunya Virus Infection
}

\author{
(DD.G. Corrêa, (D)T. A. L. Freddi, (D) H. Werner, (D)F.P.P.L. Lopes, (D) M.E.L. Moreira, (D)F.C.P. de Almeida Di Maio Ferreira,
} (D).M. de Andrade Lopes, (DF.C. Rueda-Lopes, and (D) L.C.H. da Cruz Jr

\begin{abstract}
SUMMARY: Since 2005, it has been known that mother-to-child transmission of the chikungunya virus is possible. Transmission generally occurs in the perinatal period. In the present study, we describe the brain lesions seen on MR imaging of 6 cases of perinatal chikungunya infection. Patients who underwent brain MR imaging in the acute phase presented with areas of restricted diffusion in the white matter, suggesting a perivascular distribution, whereas those in the subacute/late phase showed cystic lesions, also with a perivascular distribution, with or without brain atrophy. One patient also presented with scattered hemorrhages in the frontal and parietal lobes. Important differential diagnoses include rotavirus, Parechovirus, herpes simplex infection, and hypoxic-ischemic encephalopathy, depending on the disease phase.
\end{abstract}

ABBREVIATIONS: CHIKV = chikungunya virus; IgM = immunoglobulin M; RT-PCR = reverse transcription-polymerase chain reaction

C hikungunya is an arbovirus of the family Togaviridae, genus Alphavirus, transmitted by Aedes spp. mosquitos. In adults, the infection is characteristically manifest by fever, arthralgia, and/or cutaneous rash. ${ }^{1}$ The first documented human outbreaks were in southern Asia during the 1960s. After decades of low transmission, the virus re-emerged in 2004, causing outbreaks throughout the islands of the Indian Ocean, India, and Southeast Asia. In 2013, it was first reported in the American continent, rapidly spreading throughout 48 American countries and territories. ${ }^{2,3}$ Additionally, there are increasing reports of coinfections with other arboviruses, such as Zika and dengue, in some patients, including pregnant women, with unknown consequences. ${ }^{3}$

Studies performed during chikungunya virus (CHIKV) outbreaks reported a $4.7 \%-46 \%$ prevalence of pediatric neurologic manifestations. ${ }^{2}$ Perinatal transmission of the CHIKV was first reported during the 2005 outbreak on La Réunion Island. Essentially, neonates are asymptomatic at birth but begin to

Received August 29, 2019; accepted after revision October 2.

From the Clínica de Diagnóstico por Imagem/Diagnósticos da América (D.G.C., H.W., F.P.P.L.L., F.C.R.-L., L.C.H.d.C.), Rio de Janeiro, RJ, Brazil; Department of Radiology (T.A.L.F.), Hospital do Coração, São Paulo, SP, Brazil; and Clínica Perinatal (M.E.L.M., F.C.P.d.A.D.M.F., J.M.d.A.L.), Rio de Janeiro, RJ, Brazil.

Please address correspondence to Diogo Goulart Corrêa, MD, Clínica de

Diagnóstico por Imagem/Diagnósticos da América, Avenida das Américas, 4666,

302A, 303, 307, 325, 326, Barra da Tijuca, Rio de Janeiro, RJ, Brazil, 2640-102; e-mail:

diogogoulartcorrea@yahoo.com.br

Indicates article with supplemental on-line table.

Indicates article with supplemental on-line photo.

http://dx.doi.org/10.3174/ajnr.A6339 present with symptoms in the first days of life. ${ }^{4}$ In general, clinical manifestations of CHIKV-infected neonates are similar to those of adults: febrile illness associated with exanthema and spontaneous resolution usually observed within 1-2 weeks. ${ }^{5}$ However, up to $40 \%$ of neonates present with complicated forms of CHIKV infection, which can manifest as hemorrhagic fever, disseminated intravascular coagulation, impaired liver function, myocarditis, respiratory distress, sepsis, necrotizing enterocolitis, myocarditis, pericarditis, or neurologic compromise. ${ }^{4,5}$ Neurologic manifestations are variable and include a compromised state of consciousness, meningoencephalitis, polyneuropathy, seizures, and encephalomyelitis. ${ }^{6}$ Long-term followup of patients with perinatal CHIKV infection has shown them to have an increased risk of global neurodevelopmental delay, especially in those who experienced encephalopathy during the neonatal period. ${ }^{5}$ However, pathogenic mechanisms of the neurologic syndromes of CHIKV infection remain unknown. ${ }^{6}$

Although brain MR imaging studies of perinatal CHIKV infection have been previously described, mainly in case reports and case series, ${ }^{6-10}$ a systematic description of the lesions is still necessary to improve the understanding of its pathophysiologic mechanisms. In this article, we describe the brain MR imaging findings of 6 established cases of perinatal CHIKV infection with neurologic complications.

\section{MATERIALS AND METHODS}

The institutional review board Clinica Perinatal approved this study. The diagnosis of maternal CHIKV infection was based on the presence of serum anti-CHIKV immunoglobulin M (IgM) 


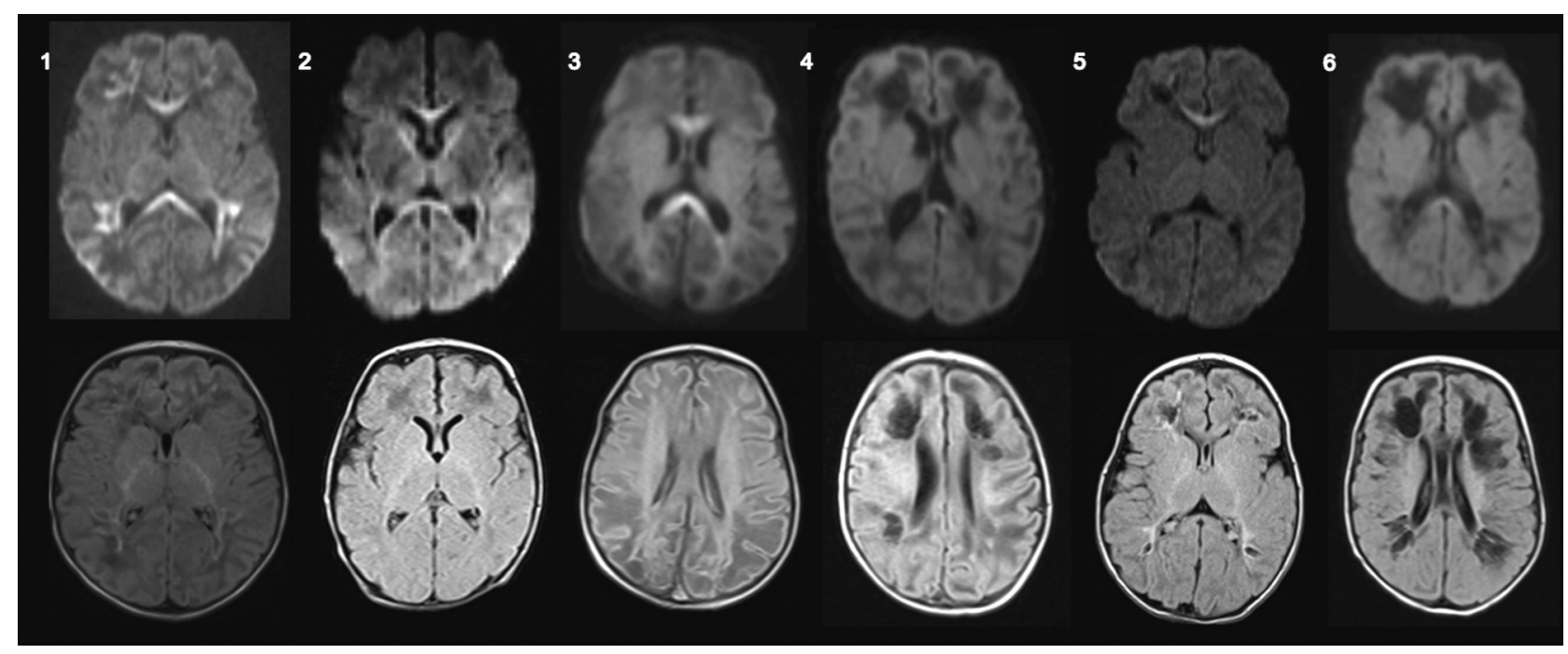

FIGURE. Brain MR imaging of 6 infants with confirmed perinatal CHIKV infection. Axial diffusion-weighted imaging (upper row) and axial FLAIR (lower row) of patients 1-6. Patients who underwent MR imaging before 14 days of symptom onset presented with restricted diffusion in the corpus callosum and/or subcortical white matter with a perivascular distribution, whereas patients who underwent MR imaging after 14 days of symptom onset presented with subcortical cystic lesions, also with a perivascular distribution with discrete restricted diffusion in the corpus callosum.

and/or serum detection of the viral genome by reverse transcription-polymerase chain reaction (RT-PCR). Mothers were screened if they presented with symptoms of CHIKV infection in the peripartum period. The diagnosis of neonatal CHIKV infection was based on the detection of serum and/or CSF antiCHIKV IgM and/or RT-PCR positive for CHIKV in the neonate. Vertical transmission was diagnosed if the mother had a serologic test positive for CHIKV in the perinatal period in association with a confirmed infection of the symptomatic neonate within the first 21 days of life. Detection of anti-CHIKV IgM was performed by IgM capture enzyme-linked immunosorbent assay. All participants were also tested for the Zika virus, dengue virus, toxoplasmosis, cytomegalovirus, rubella, herpes virus, syphilis, and human immunodeficiency virus.

Brain MRIs were performed on 1.5T clinical scanners (Avanto, Aera, and Espree; Siemens, Erlangen, Germany). The MR imaging protocol included axial T1-weighted images with or without intravenous injection of a gadolinium-based contrast agent, axial and coronal T2-weighted images, FLAIR, axial diffusion-weighted imaging with apparent diffusion coefficient maps, and axial SWI. No participant underwent spine MR imaging.

\section{Case Series}

From 8 cases of confirmed CHIKV vertical transmission, 2 presented with normal brain MR imaging findings and 6 had brain lesions detected by MR imaging. In all cases, the mothers presented with typical symptoms of CHIKV infection in the peripartum period (On-line Table).

Case 1. A 5-day-old neonate with a reduced level of consciousness. A hemogram showed severe thrombocytopenia. Brain MR imaging on the 15th day showed areas of restricted diffusion in the entire corpus callosum and subcortical white matter of the frontal, parietal, and temporal lobes, suggesting perivascular distribution, with discrete hyperintense signal on FLAIR (Figure and On-line Table).

Case 2. A 10-day-old neonate presented with sepsis and seizures. Brain MR imaging on the 20th day of life showed areas of restricted diffusion in the entire corpus callosum and white matter of the frontal, parietal, and temporal lobes. FLAIR, T1-, and T2-weighted imaging findings were unremarkable (Figure and On-line Table).

Case 3. A 5-day-old neonate presented with fever and a reduced level of consciousness. Brain MR imaging on the 18th day of life showed bilateral subcortical white matter lesions with hypointense signal on T1-weighted imaging and FLAIR and hyperintense signal on T2-weighted imaging, associated with restricted diffusion in the entire corpus callosum. SWI showed hemorrhage in the right frontal and parietal lobes (Figure, On-line Table, and On-line Figure).

Case 4. A 7-day-old neonate presented with fever and irritability and had 1 seizure. Brain MR imaging in the 21st day of life showed diffuse brain volume loss with enlarged ventricles and bilateral cystic lesions, suggesting a perivascular distribution, and a small area of restricted diffusion in the splenium of the corpus callosum (Figure and On-line Table).

Case 5. A 4-day-old neonate presented with fever and 2 episodes of seizures. Brain MR imaging on the 20th day of life showed small subcortical cystic lesions in the frontal and parietal lobes, suggesting a perivascular distribution, and restricted diffusion in the genu of the corpus callosum (Figure and On-line Table).

Case 6. A 4-day-old neonate presented with fever and seizures. Brain MR imaging on the 30th day of life showed brain volume loss, enlarged ventricles, as well as subcortical cystic lesions in the frontal and parietal lobes, also suggestive of perivascular

AJNR Am J Neuroradiol 41:174-77 Jan 2020 www.ajnr.org 
distribution, and a discrete restricted diffusion in the splenium of the corpus callosum (Figure and On-line Table).

All infants had normal findings on prenatal ultrasonography follow-up and were born without complications. All participants had serology negative for HIV and herpes and laboratory tests negative for syphilis. The participants also had IgM antibodies negative for Zika, dengue, cytomegalovirus, and rubella viruses. No participant was excluded because of coinfections. Blood and/ or CSF samples of the neonates tested positive for CHIKV as assessed by RT-PCR.

\section{DISCUSSION}

We reported brain abnormalities as measured by MR imaging in 6 confirmed cases of vertical transmission of the chikungunya virus. All participants were younger than 1 month of age at the time of MR imaging, were born asymptomatic, and developed symptoms between 4 and 10 days of life. Imaging findings can be divided in 2 patterns: those imaged within 2 weeks of disease onset, here designated as the early phase, presenting with areas of restricted diffusion in the subcortical white matter of both cerebral hemispheres and in the corpus callosum; and those imaged $>2$ weeks after disease onset, designated as subacute/delayed phase, presenting with bilateral cystic lesions with or without brain atrophy and hemorrhage.

The mechanisms through which CHIKV affects the CNS have not been fully elucidated, and it is still unclear whether the virus acts directly by targeting neurons and glial cells or indirectly by triggering immune-mediated effects through upregulation of inflammatory and antiviral cytokines. ${ }^{3,11}$ In experimental studies, both ribonucleic acid and the virus itself have been isolated from CSF, suggesting direct neuroinvasion, ${ }^{12}$ but brain postmortem examinations with histopathologic analyses were unable to detect CHIKV. ${ }^{11,13}$ Hence, the target cells of CHIKV in the human brain remain unknown. Studies in human cells demonstrated the susceptibility of neuroblasts and glial cells, such as astrocytes and microglia, to CHIKV invasion. The apoptosis observed in $\mathrm{CHIKV}$-infected neuroblasts and astrocytes suggests a direct role of viral infection in disease pathogenesis. ${ }^{11}$ On the other hand, concentrations of tumor necrosis factor- $\alpha$, interferon- $\alpha$, interleukin-6, and monokines induced by interferon- $\gamma$ were found to be significantly higher in patients with neurologic disease secondary to CHIKV, as opposed to uncomplicated infections. ${ }^{3}$ Direct viral CNS infection is believed to be more probable in neonates and the elderly due to their weaker innate and adaptive immune responses. This belief is supported by the high rate (92\%) observed of CHIKV RT-PCR positivity in infected neonates' CSF and the short latency between initial CHIKV-fever signs and encephalitic symptoms. ${ }^{14}$

In addition, microgliosis and perivascular lymphocytic infiltrates were found in the brain parenchyma of those infected by CHIKV. ${ }^{13,15}$ Couderc et al, ${ }^{16}$ studying mouse models with CHIKV infection, found that cells in the choroid plexus, ependymal wall, and leptomeninges, including external cells in the Virchow-Robin spaces, were strongly infected with CHIKV, whereas those in the brain parenchyma were not. Thus, in their experimental study, despite strong infection of the meninges and Virchow-Robin spaces, the barrier function of the brain microvessels was preserved. Leptomeningeal cells form an interconnected multicellular network, which acts as a regulatory interface between CSF and the brain surface, as well as among arterioles and the surrounding neural tissue (perivascular spaces), and this tissue organization and physiology may play a role in CHIKV dissemination. ${ }^{16}$ Our study showed that regardless of the pathophysiology, CHIKV encephalitis can produce serious brain lesions with potential consequences for neural development. Furthermore, some patients presented with restricted diffusion or cystic lesions along the course of the deep medullary veins, suggesting perivascular involvement.

Although early vertical transmission has been demonstrated, ${ }^{17}$ symptomatic neonatal disease generally occurs from intrapartum maternal infections. ${ }^{3}$ Mother-to-child transmission of CHIKV is relatively rare (approximately $2.5 \%$ of exposed neonates become infected), but the intrapartum transmission rate of viremic women is close to $50 \%$, highlighting this period as critical for disease transmission. Previous observations have suggested that the placental barrier is relatively effective in preventing antepartum CHIKV transmission. In contrast, vertical transmission is frequently observed in viremic mothers at the end of pregnancy, when the maternal blood can come into contact with placental barrier breaches resulting from uterine contractions during labor. High viral load measured in placentas from infected neonates is thus most likely a consequence of elevated maternal viremia. ${ }^{7}$ These data are in accordance with our results; although we did not investigate the viral presence in placentas of our participants, all neonates were born asymptomatic and developed symptoms within the first days of life. In addition, none of our participants presented with evidence of mosquito bites.

Among our cases, 4 neonates were born by cesarean delivery and 2 were born by vaginal delivery. A cesarean delivery does not appear to prevent vertical transmission; therefore, systematic performance of cesarean deliveries for infected mothers to reduce the risk of viral transmission is not recommended. ${ }^{3,18}$ Additionally, in a study by Torres et al, ${ }^{18}$ cesarean delivery was unrelated to mother-to-child CHIKV transmission. The ideal type of delivery and the interval between symptom onset and childbirth are still unknown.

Previous authors have found that at the acute stage (within 2 weeks of disease onset), brain MR imaging of perinatal CHIKVinfected children revealed scattered restricted diffusion in the subcortical white matter and corpus callosum, with unremarkable features on T1- and T2-weighted imaging. ${ }^{6-10}$ Restricted diffusion is related to cytotoxic edema and may be secondary to plasma leakage from capillaries and venules associated with immune-mediated allergic perivascular demyelination. ${ }^{13}$ In the subacute phase (15-45 days after disease onset), the areas that exhibited low signal on diffusion-weighed imaging were related to vasogenic edema. In the chronic phase ( $>45$ days after disease onset), the lesions evolved toward cavitations and parenchymal atrophy. ${ }^{6-10}$ Foci of intraparenchymal hemorrhage scattered through white matter have also been described. ${ }^{8,10}$ Although we did not perform an imaging follow-up of our patients, our results show similarities to previous studies because patients who were studied early in the disease course presented with white matter- and corpus callosum-restricted 
diffusion, whereas those in the late phases showed cystic lesions and brain atrophy. Most interesting, we found an association between subcortical cystic lesions and restricted diffusion in some participants. This may be because cells of the corpus callosum have a higher density of cytokine, glutamate, and other excitatory amino acid receptors, compared with other brain areas, resulting in a tendency for developing cytotoxic edema. ${ }^{19}$

The main differential diagnoses for neonatal brain CHIKV infection in the acute phase are rotavirus and parechovirus infections, which have similar imaging patterns, though most cases of rotavirus encephalitis are asymptomatic. ${ }^{20,21}$ In the case series of Sarma et $\mathrm{al}^{21}$ of neonatal parechovirus meningoencephalitis, 1 patient underwent a longitudinal follow-up at 3 months and did not present with cystic lesions. This finding is in marked contrast to those of our patients with perinatal chikungunya. However, other authors described the development of extensive cystic leukomalacia at the 4-month follow-up in a patient with parechovirus infection. ${ }^{21}$ Some data suggest that preterm neonates are at higher risk of severe cystic leukoencephalomalacia in parechovirus infection. ${ }^{21}$ Whether prematurity is also a risk factor for the development of cystic encephalomalacia in perinatal CHIKV infection needs further investigation. In the chronic phase, differential diagnoses for cystic lesions include herpes simplex infection and hypoxic-ischemic encephalopathy. ${ }^{20-22}$ CHIKV-related lesions with perivascular dissemination could aid in the differentiation. However, the real value of this type of lesion distribution needs further research.

Our study has some limitations. First, we did not perform follow-up evaluations to confirm whether all patients who presented with restricted diffusion in the acute phase would evolve to brain cavitation and atrophy, or assess how the cavitations would evolve, or evaluate the neurologic outcome of the participants. Also, we did not perform amniocentesis or placental biopsies to search for the virus before birth. However, all participants had a confirmed diagnosis of CHIKV infection, and other coinfections or peripartum complications were excluded.

\section{CONCLUSIONS}

Neuroimaging can aid in the diagnosis of perinatal CHIKV infection. Bilateral subcortical restricted diffusion and cystic lesions with or without brain atrophy with a perivascular distribution are characteristic findings, dependent on the disease stage. However, whether this perivascular distribution can aid in the differential diagnosis needs further research.

Disclosures: Maria E.L. Moreira-UNRELATED: Employment: Fiocruz*; Payment for Manuscript Preparation: Nestle do Brasil, review article about milk osmolarity.* Fernanda C. Rueda-Lopes-UNRELATED: Employment: Diagnósticos da América. *Money paid to the institution.

\section{REFERENCES}

1. Contopoulos-Ioannidis D, Newman-Lindsay S, Chow C, et al. Mother-to-child transmission of Chikungunya virus: a systematic review and meta-analysis. PLoS Negl Trop Dis 2018;12:e0006510 CrossRef Medline
2. Almeida Bentes A, Kroon EG, Romanelli R. Neurological manifestations of pediatric arboviral infections in the Americas. J Clin Virol 2019;116:49-57 CrossRef Medline

3. Mehta R, Gerardin P, de Brito CA, et al. The neurological complications of Chikungunya virus: a systematic review. Rev Med Virol 2018;28:e1978 CrossRef Medline

4. Barr KL, Vaidhyanathan V. Chikungunya in infants and children: is pathogenesis increasing? Viruses 2019;11 CrossRef Medline

5. Cardona-Correa SE, Castaño-Jaramillo LM, Quevedo-Vélez A. Vertical transmission of Chikungunya virus infection: case report. Rev Chil Pediatr 2017;88:285-88 CrossRef Medline

6. Bandeira AC, Campos GS, Sardi SI, et al. Neonatal encephalitis due to Chikungunya vertical transmission: first report in Brazil. IDCases 2016;5:57-59 CrossRef Medline

7. Gérardin P, Barau G, Michault A, et al. Multidisciplinary prospective study of mother-to-child Chikungunya virus infections on the island of La Réunion. PLoS Med 2008;5:e60 CrossRef Medline

8. Ramful D, Carbonnier M, Pasquet M, et al. Mother-to-child transmission of Chikungunya virus infection. Pediatr Infect Dis J 2007; 26:811-15 CrossRef Medline

9. Gérardin P, Sampériz S, Ramful D, et al. Neurocognitive outcome of children exposed to perinatal mother-to-child Chikungunya virus infection: the CHIMERE cohort study on Reunion Island. PLOS Negl Trop Dis 2014;8:e2996 CrossRef Medline

10. Karthiga V, Kommu PP, Krishnan L. Perinatal Chikungunya in twins. J Pediatr Neurosci 2016;11:223-24 CrossRef Medline

11. Matusali G, Colavita F, Bordi L, et al. Tropism of the Chikungunya virus. Viruses 2019;11:175 CrossRef Medline

12. Tandale BV, Sathe PS, Arankalle VA, et al. Systemic involvements and fatalities during Chikungunya epidemic in India, 2006. J Clin Virol 2009;46:145-49 CrossRef Medline

13. Ganesan K, Diwan A, Shankar SK, et al. Chikungunya encephalomyeloradiculitis: report of 2 cases with neuroimaging and 1 case with autopsy findings. AJNR Am J Neuroradiol 2008;29:1636-37 CrossRef Medline

14. Cerny T, Schwarz M, Schwarz U, et al. The range of neurological complications in Chikungunya fever. Neurocrit Care 2017;27:44757 CrossRef Medline

15. Pinheiro TJ, Guimarães LF, Silva MTT, et al. Neurological manifestations of Chikungunya and Zika infections. Arq Neuropsiquiatr 2016;74:937-43 CrossRef Medline

16. Couderc T, Chrétien F, Schilte C, et al. A mouse model for Chikungunya: young age and inefficient type-I interferon signaling are risk factors for severe disease. PLoS Pathog 2008;4:e29 CrossRef Medline

17. Touret Y, Randrianaivo H, Michault A, et al. Early maternal-fetal transmission of the Chikungunya virus. Presse Med 2006;35:165658 CrossRef Medline

18. Torres JR, Falleiros-Arlant LH, Dueñas L, et al. Congenital and perinatal complications of Chikungunya fever: a Latin American experience. Int J Infect Dis 2016;51:85-88 CrossRef Medline

19. Starkey J, Kobayashi N, Numaguchi Y, et al. Cytotoxic lesions of the corpus callosum that show restricted diffusion: mechanisms, causes, and manifestations. Radiographics 2017;37:562-76 CrossRef Medline

20. Yeom JS, Kim YS, Seo JH, et al. Distinctive pattern of white matter injury in neonates with rotavirus infection. Neurology 2015;84:2127 CrossRef Medline

21. Sarma A, Hanzlik E, Krishnasarma R, et al. Human parechovirus meningoencephalitis: neuroimaging in the era of polymerase chain reaction-based testing. AJNR Am J Neuroradiol 2019;40:1418-21 CrossRef Medline

22. Verboon-Maciolek MA, Groenendaal F, Hahn CD, et al. Human parechovirus causes encephalitis with white matter injury in neonates. Ann Neurol 2008;64:266-73 CrossRef Medline 